\title{
Acute Tonsillitis and Bronchitis in Russian Primary Pediatric Care: Prevailing Antibacterial Treatment Tactics and Their Optimization
}

\author{
Vladimir Tatochenko ${ }^{1, ~ *}$, Eugenia Cherkasova ${ }^{2}$, Tatjana Kuznetsova ${ }^{3}$, Diana Sukhorukova ${ }^{4}$, \\ Maya Bakradze ${ }^{5}$ \\ ${ }^{1}$ National Medical Research Centre of Child Health, Moscow, Russia \\ ${ }^{2}$ Pulmonology and Allergology Department, S. I. Kruglaya Clinical Research Centre, Oryol, Russia \\ ${ }^{3}$ Internal Disease Department, Medical College, I. S. Turgenev State University, Oryol, Russia \\ ${ }^{4}$ City Pediatric Polyclinic No.4, Medical College, I. S Turgenev State University, Oryol, Russia \\ ${ }^{5}$ Diagnostic Department, National Medical Research Centre of Child Health, Moscow, Russia
}

Email address:

tatovk@yandex.ru(V. Tatochenko)

${ }^{*}$ Corresponding author

\section{To cite this article:}

Vladimir Tatochenko, Eugenia Cherkasova, Tatjana Kuznetsova, Diana Sukhorukova, Maya Bakradze. Acute Tonsillitis and Bronchitis in Russian Primary Pediatric Care: Prevailing Antibacterial Treatment Tactics and Their Optimization. American Journal of Pediatrics.

Vol. 4, No. 3, 2018, pp. 46-51. doi: 10.11648/j.ajp.20180403.11

Received: May 25, 2018; Accepted: June 27, 2018; Published: July 26, 2018

\begin{abstract}
Inappropriate use of antibiotics in children with acute tonsillitis (AT) and bronchitis is an important cause of the microbial resistance. The aim of the study was to find out pediatricians' motives in prescribing antibiotics and the extent of their inappropriate use in these cases, as well as maternal attitudes to the use of antibiotics in acute viral respiratory infections (ARI). We also studied in the context of regular primary pediatric care the acceptability to parents of a judicious use of antibiotics. Pediatricians ( $\mathrm{n}=97)$ attitudes to antibiotics and their practices were studied by a questionnaire in 4 cities of Russia, mothers' attitudes to antibiotics $(\mathrm{n}=107)$ and antibiotic use frequencies were studied in an Oryol polyclinic. Optimization of treatment studies (acute tonsillitis 1577 child-years, acute bronchitis 3303 child-years of observations) were conducted by two co-authors in their capacity of primary pediatric providers (for about a 1000 children each) in a polyclinics of Oryol. Antibiotics were given only to AT cases found positive for hemolytic streptococcus (GABHS) by an express-test. Patients with acute bronchitis and bronchiolitis were treated by inhalation of $0.9 \%$ or $3 \%$ saline solutions. Most (95-100\%) pediatricians consider tonsillitis (without bacteriological evidence obtained at the point of care) to be of bacterial origin and treat it with antibiotics - for the fear of GABHS complications, self-protection or as a traditions. About a half of mothers would add antibiotics to whatever else pediatrician prescribes for ARI, 1/3 mothers prefer self-treatment with antibiotics if body temperature is over $38^{\circ} \mathrm{C}$. Of tonsillitis patients $80.5 \%$ and of bronchitis over $70 \%$ - are treated in the outpatient setting with antibiotics. The incidence of tonsillitis was 80 per 1000 children of all ages, the proportion of GABHS-tonsillitis - $27 \%$ - only in children above 4 years of age (population incidence 7.5 per 1000). 87\% of mothers of GABHS negative children accepted treatment without antibiotics. Similarly, $88.5 \%$ of parents of children with bronchiolitis (incidence 113 per 1000 0-2 years old) and acute bronchitis (61 per 1000 0-18 years old) agreed to use only saline inhalations. We conclude that additional attention to mothers (explaining results of the express-test, or teaching how to do inhalation) overcome their fears of withholding antibiotics.
\end{abstract}

Keywords: Acute Tonsillitis, Bronchiolitis, Acute Bronchitis, Children, Antibiotics. Pediatricians' Views and Perceptions, Maternal Attitudes 


\section{Introduction}

Development of antibiotic resistance by pathogens causing community acquired infection is to a considerable degree linked to inappropriate use of antibiotics in pediatric practice [1]. Of particular importance is an excessive use of antibiotics for treatment of acute viral respiratory infections (ARI), and among them acute tonsillitis (AT) ${ }^{1}$, acute bronchitis and bronchiolitis - in spite of recommendations in international and national guidelines to limit antibiotics use [2-5]. It reflects the fear of some pediatricians to miss cases of AT caused by group A ß-hemolytic streptococci (GABHS) that - if untreated - could cause both local suppuration and complications such as acute rheumatic fever, chorea minor, glomerulonephritis etc. An excessive prescriptions of antibiotics may reflect the wish to start treatment before the results of the throat culture (if any) are known [6].

The high incidence of bronchitis (in Russia 75-200, in the USA 56 per 1000 children per year $[7,8]$ ) cause $25-40 \%$ of all pediatric primary care visits [9]. Frightening for many parents manifestations (fever, cough, wheezing) raise the demand for antibiotics though practically all of them are caused by respiratory viruses [4]. Bacteria cause bronchitis in children in about $5 \%$ of cases (mostly in school age), they are mainly Mycoplasma pneumoniae and Chlamydia pneumoniae $[6,8,10,11]$. Hence antibiotics have no place in the majority of bronchitis and bronchiolitis cases, and it is proven by a number of conclusive studies [10, 11], and recommendations to this effect are found in international and national guidelines $[3,5,13]$. Nevertheless antibiotics are widely used in bronchitis - in Europe in $40-67.5 \%$ [4, 5, 12, 13]. In the USA up to $1 / 3-1 / 2$ of antibiotics in the lower respiratory tract infections considered to be used inappropriately $[14,15]$.

In Russia we have a similar picture. Pharmaco-economic study in a regional pediatric hospital showed that $85 \%$ of children with bronchitis would get antibiotics, in 30\% - 2 courses; antibiotics took the biggest share of total costs [16]. An audit program of treatment modalities in 21 pediatric hospitals in 4 regions of Russia had shown that $64-77 \%$ of all children with fever (the majority of them with ARI) are treated with antibiotics (in $85 \%$ the parenteral ones). Practically all children with bronchitis and bronchiolitis get antibiotics, in $95 \%$ of them the $3 \mathrm{~d}$ generation cephalosporins, the rest - aminoglycosides, carbopenems, cefepim [17]. Similar situation was found in 7 pediatric hospitals in 2 additional regions audited by one of co-authors ( $\mathrm{MB}$ - to be published).

Our aim was to study the prevailing practices of antibiotic use in acute tonsillitis and bronchitis by pediatricians, as well as parental attitudes to antibiotics for ARI. We also studied the acceptability of a recommended judicious use of antibiotics for this conditions by parents in the context of regular practices of primary care by (E. C. and D. S.) as

1 Acute inflammation of tonsils and surrounding mucous membranes often referred to as acute tonsillo-pharingitis. ouchastok pediatricians working in a city of Oryol polyclinic $^{2}$.

\section{Material and Methods}

To study pediatricians' knowledge about antibiotic treatment of AT we conducted by questionnaire to 97 pediatricians in the cities of Oryol, Archangelsk, Vladivostok and Petropavlovsk-Kamchatsky. We also conducted a survey of attitudes and practices with respect to antibiotics in 107 mothers in an Oryol polyclinic.

In AT treatment study were included all children in one ouchastok of a pediatric polyclinics of Oryol cared for by one co-author (EC) for 2 years (1577 child-years of observations). All 140 cases of AT diagnosed were tested with their mothers present by an express-test for $\mathrm{GABHS}^{3}$; the test had been previously compared to culture and found by us to be $99 \%$ concordant [18]. Antibiotics were given only to patient with a positive test, parents of children negative for GABHS were persuaded to accept only symptomatic treatment for their children.

The bronchitis and bronchiolitis study was also conducted in one polyclinic ouchastok in Oryol cared for by one of coauthors (DS) for 3 years (3303 child-years of observations). All children with bronchiolitis (51) and acute bronchitis (142 with 202 episodes) were included. Their treatment regiments included sodium chloride solutions $(0.9$ or $3 \%)$ inhalations without antibiotics ${ }^{4}$. All parents were instructed how to perform saline inhalations at the time of diagnosis by pediatrician or the nurse; they were warned of a possible increase in coughing after the first inhalation of $3 \%$ saline. The treatment results were compared to those in 3 groups of similar patients at the adjacent ouchastok treated with systemic antibiotics and/or a broncholytic (fenoterol + ipratropium bromide diluted by normal saline) inhalations.

\section{Results}

\subsection{Attitudes of Pediatricians to $A T$}

A survey conducted in 4 cities of Russia showed considerable lacunae in pediatricians' knowledge of ethology of AT and of indications for antibiotics [19]. Independent of the region, in- or outpatient place of work and the length of practice 85 to $100 \%$ of pediatricians would treat AT with antibiotics - considering bacterial infection to be an obligatory element of AT etiology. Similarly the majority of pediatricians answered that they would give antibiotic for "a preventive purpose" (meaning, rather self-overprotection "to be on a safe side"). As a motive appealing to the

2 Pediatric polyclinic in Russia provides primary health and medical care, each pediatrician supported by a nurse serving about 1000 children (age 0-18 years) in the neighborhood called "ouchastok" (a town quarter).

3 Streptatest, Laboratoires Dectra Pharm \& Sbh, France.

$43 \%$ sodium chloride inhalations hads shown promising result in the treatment of bronchitis in the National Medical Centre for Childrens' Health and elswhere [20]. 
traditions of a medical establishment was more characteristic $(93 \%)$ to pediatricians with less than 5 years in service due to their relative lack of independence; for those over 15 years in service $(88 \%)$ such practice means just to follow a stable stereotype.
Fear of reprimands for "insufficiently intensive therapy" without antibiotics was admitted by $93 \%$ of doctors with less than 5 , and by $61 \%$ with $5-10$ years in practice; the frequency of this motive rapidly decreases with increasing years in practice - to $7 \%$.

Table 1. Tonsillitis' signs and symptoms considered by in- and outpatient pediatricians as indication for antibiotic therapy (\%).

\begin{tabular}{|c|c|c|c|c|}
\hline \multicolumn{3}{|l|}{ Signs and symptoms } & \multirow{2}{*}{$\begin{array}{l}\text { Outpatient } \mathbf{n}=\mathbf{7 7} \\
97.4\end{array}$} & \multirow{2}{*}{$\begin{array}{l}\text { Inpatient } \mathbf{n}=\mathbf{2 0} \\
95.0\end{array}$} \\
\hline Tonsils with visible fo & exudate $\mathrm{o}$ & & & \\
\hline \multirow{5}{*}{ Thro at redness, T0C } & \multirow{2}{*}{$37-38^{\circ} \mathrm{C}$} & $1-2$ dais & $49.4^{*}$ & $85.0^{*}$ \\
\hline & & $>3$ dais & 80.5 & 75.0 \\
\hline & \multirow{2}{*}{$38-39^{\circ} \mathrm{C}$} & 1-2 dais. & 81.8 & 95.0 \\
\hline & & $>3$ dais & 89.6 & 100.0 \\
\hline & \multirow[t]{2}{*}{$>39^{\circ} \mathrm{C}$} & $\geq 1$ days. & 96.1 & 100.0 \\
\hline Red conjunctivae & & & 32.5 & 55.0 \\
\hline
\end{tabular}

$* \mathrm{P}=0.003$

Respondents had been asked about symptoms that prompt them to prescribe antibiotics. Results are shown in tabl.1. Pediatricians of any length of practice considered AT to be bacterial if patients had:

1. red throat with follicles, exudate or a film on tonsils plus $\mathrm{T} 39^{\circ} \mathrm{C}$ (practically all doctors);

2. AT with the temperature above $37.5^{\circ} \mathrm{C}$ (over one half);

3. AT with conjunctivitis (about one third);

4. AT with aphthous stomatitis (71\% - 100\% of respondents).

The only significant difference was found in patient with 1-2 days long subfebrile temperature - in hospitals they are twice as likely to be given antibiotics. There were some regional differences with respect to conjunctivitis - it was considered as a sign of bacterial infection by $78 \%$ of Archangelsk and the Far east cities and only by $7.5 \%$ in Oryol $(\mathrm{P}=0.001)$..

This study has clearly shown that pediatricians were not following guidelines repeatedly published in Russia and abroad - apparently since without bacteriological evidence of the AT etiology obtained at the point of care the majority of AT patients would be started on antibiotics as dictated by the fear of GABHS-AT complications and/or reprimands from administration, or by the institutions' tradition.

\subsection{Maternal Attitudes Towards Antibiotics for ARI}

A survey of maternal attitude to antibiotics for their children was conducted by us parallel with AT study. About a half of 107 mothers interviewed admitted that they would add antibiotics to whatever else pediatrician prescribes for ARI, 1/3 of mothers prefer self-treatment giving antibiotics based on their own experience or on advice of relatives and neighbors. Temperature over $38^{\circ} \mathrm{C}$ was considered to be an indication for antibiotics by $33 \%$ of mothers interviewed.

\subsection{Reducing Antibiotics for Acute Tonsillitis}

The analysis we conducted on another ouchastok of our polyclinic that followed an old traditions of AT treatment showed that of 135 AT cases antibiotics were given to 108 $(80.5 \%)$.

We included in our study all children with AT from one ouchastok for 2 years seen personally by one of co-authors (EC) (Table 2). To diagnose GABHS-AT we express tested all children diagnosed with AT.

Table 2. Acute tonsillitis in children 0-18 years old on one pediatric ouchastok (1755 person years of observation)*

\begin{tabular}{|c|c|c|c|c|c|c|c|}
\hline Age & 6-11 mo & $1-3 y$ & 4-6 y & $7-10 y$ & 11-14 y & $15-18 y$ & Total \\
\hline Number of AT/Population & $7 / 117$ & $85 / 283$ & $31 / 275$ & $7 / 373$ & $7 / 393$ & $3 / 314$ & $140 / 1755$ \\
\hline Incidence per 1000 & 82 & 341 & 78 & 15 & 10 & 7 & 84 (95\% CI: 71-97) \\
\hline Number of GABHS-AT/all AT & - & - & \multicolumn{4}{|c|}{$13 / 48(27 \%)^{* *}$} & $13 / 140(9.2 \%)$ \\
\hline
\end{tabular}

* two cases of PFAPA-syndrome were excluded.

** including 3 cases of scarlet fever.

AT was diagnosed in $9.7 \%$ of children with ARI with seasonal picks in February - March and September in both years of observation when most cases of GABHS-AT occurred. GABHS-AT was not found in children below 4 years of age, 13 cases were diagnosed in children 4-18 years of age - that is in $27 \%(95 \% \mathrm{CI}: 14.5-41.5 \%)$ of AT in this age group. (Of AT in all age groups GABHS-AT were found in $9.2 \%(95 \% \mathrm{CI}: 7.8-41.5 \%)$ with population incidence of 7.5 per 1000 (95\% CI: 3.2-11.6).

Of children tested negative for GABHS infectious mononucleosis was diagnosed in 6 (by PCR for EBV), adenovirus (by RIF) - in 21 of 56 children tested.

We used our data to test the diagnostic value of $\mathrm{W}$. McIsaac's scale [21], All GABHS-AT children had scores above 2 that gives sensitivity of $100 \%$. However only $8 \%$ of children with viral AT had the score $0-1,12 \%$ scored $2-3$ and $80 \%-\geq 4-$ it corresponds to specificity of only $7.7 \%$.

All 13 children with GABHS-AT were successfully treated with amoxicillin for 10 days. Among 127 children with viral AT antibiotics were given by us to 5 with otoscopic feature 
of otitis media and to one who recently had a cardiac surgery. On insistence of parents 8 children were started on antibiotics, but after 48 hours seeing no effects they yielded to pediatrician's advice to stop them. Parents of three patients (two of them with infectious mononucleosis) asked to be referred to a hospital where they traditionally were given antibiotics. Thus, out of 127 patients in the viral AT group we could withheld antibiotics in $111(87 \%)$. In total antibiotics were given to 29 patients of $140(21 \%)-4$ times less than $80.5 \%$ found in our survey of other ouchastoks of the same polyclinic.

The small number of mothers that hesitated to stay off antibiotics for their children with the negative express test was unexpected given the results of our survey. Apparently on the spot diagnosis of non-GABHS-AT is convincing for a majority, and those who hesitated to accept it soon found that antibiotics were not effective and agreed to drop them.

\subsection{Reducing Antibiotics for Acute Bronchitis and Bronchiolitis}

This 3 year study was aiming at minimizing the use of antibiotics for both bronchiolitis and acute bronchitis by providing symptomatic treatment that included inhalations of sodium chloride $0.9 \%$ or $3 \%$ solutions at home. The first inhalation was conducted under the instruction of the pediatrician at the time of diagnosis. During 3 years we diagnosed 51 case of bronchiolitis - the population incidence 108 (95\% CI: 80-136) per 1000 0-2 years old children, We also diagnosed 202 episodes of bronchitis in 142 children (61 (95\% CI: 53-69) per 1000 0-18 years old) of which 75 children had 108 wheezing episodes - 33 (95\% CI: 27-39) per 1000. Among patients with bronchitis $75 \%$ were children below 5 years of age, and among wheezing ones - $60 \%$.

Of 51 patients with bronchiolitis 10 with the $\mathrm{SaO}_{2}<93 \%$ and/or severe dispnoe were sent to hospital, 41 were treated at home randomly divided to receive inhalations of normal saline (19) or $3 \%$ sodium chloride solution (21). The results (published previously [22]) were compared to a similar group treated with antibiotics and/or a bromcholytic showed that antibiotics were inferior to inhalations:

1. of $3 \%$ saline with regards to the duration of tachypnea and dyspnea, $\mathrm{O}_{2}$ desaturation and the time of rales' clearance on auscultation,

2. of $0.9 \%$ saline inhalations - to the duration of dyspnea.

3. of broncholitic inhalation - to the duration of tachypnea and dyspnea.

Inhalation of broncholytics had no statistically valid advantages to either saline inhalation on all above counts.

Of 202 episodes of acute bronchitis we gave antibiotics to 10 children with otitis media and UTI; in 17 school children we suspected $M$. pneumonia infection (confirmed in 5 cases by PCR) and gave macrolides with quick clinical effect. Parents of only 3 wheezing children asked to be referred to a hospital where they were given antibiotics. Thus antibiotics were given to $15 \%$ of patients, inappropriately - in 3 of 175 episodes - about $1.5 \%$ ).

The rest of 172 episodes of bronchitis were treated at home; non-wheezing episodes (78) were treated by inhalations of normal saline, those with wheezing (94) - with 3\% sodium chloride solution. Results were compared to those on an adjacent ouchastok where antibiotics were given to 70 patients (in 21 with wheezing - plus a bronchodilator) whereas 23 patients with normal temperature were given only bronchodilator inhalations. We found that percentage of children with bronchitis treated with antibiotics in this ouchastok (about 70\%) is representative for other polyclinic pediatricians that would withhold antibiotics only to patients with normal temperature.

Statistical analysis had shown that antibiotics provide no advantages as compared to saline solution inhalations independent of the criteria used (duration of fever, tachypnea and the time of rales' clearance on auscultation). Inhalation of 3\% saline alone was superior to bronchodilator (with antibiotics or alone) when evaluated for the velocity of the rales' clearance.

\section{Discussion}

Our study (as a similar by I. Dronov, [23]) shows that pediatricians in Russia - young or experienced, no matter in what region or in in- or outpatient settings, would treat $85 \%$ and more AT cases with antibiotics since they consider bacteria as obligatory agents of its etiology. Most of them consider tonsillar exudate and red throat with an elevated temperature as a sign of bacterial inflammation. These situation, whatever motives pediatricians claim in not following guidelines, is clearly due to non-availability till very recently of a convenient express test for GABHS. Laboratories for throat culture, particularly in outpatient setting are not easily accessible, and even if so, the results come 1-2 days later and antibiotics in the meantime seem to be a good practice. Moreover, laboratories instead of giving "negative for GABHS" result often mention commensal bacteria grown (such as Staphylococci, Streptococcus viridnce) that may support the idea of AT bacterial etiology. Introduction of express tests should change the situation.

Our experience with an express test shows that its application on the spot of care influences the decision of mothers whose high acceptance of non-antibiotic treatment of AT was at discordance with their reported attitudes to antibiotics.

Though express tests for GABHS are not very expensive (about $\$ 4$ each) with a high incidence of AT (84 per 1000) and a low proportion (9.2\%) of GABHS-AT among them the total cost of testing on 1 ouchastok of 1000 children per year may come up to $\$ 320$. Our data shows that testing children below 4 years of age is not necessary, in older children those with 0-1 score on W. McIsaac's scale could also be excluded. Our calculations show that with this approach economy on antibiotics by far exceeded the cost of testing.

Attitudes towards bronchitis and bronchiolitis being a bacterial infection that require antibiotics in Russia are prevailing among pediatricians as it is in the case of AT. In the real practice only $20-30 \%$ of children with bronchitis - those 
with normal temperature may escape an antibiotic prescription. There is no express test to select cases of bronchitis of bacterial origin, routine throat and sputum cultures often grow $S$. pneumoniae and $H$. influenzae, that does not help pediatricians to abstain from antibiotics. Clinical features of $M$. pneumonia allow to diagnose these cases (due to a high carriage rate PCR and serology may only increase false positivity). Our experience with saline solutions' inhalation therapy allowed to limit antibiotics to $15 \%$ of all cases. Moreover we demonstrated advantages of 3\% saline solution inhalations to use of a broncholytic in wheezy bronchitis.

Success in persuading mothers to abstain from antibiotics (as in the case of no antibiotics for non-GABHS AT) was to a considerable degree a result of closer than at a routine visit interactions with the pediatrician in the process of inhalation instructions. Positive results of the treatment provided support to mothers in the acceptance of "no antibiotics for bronchitis" offered by doctor with appropriate explanations.

\section{Conclusion}

A high (70-80\%) antibiotic prescription rate for acute tonsillitis and bronchitis in primary pediatric care often result from "to be on a safe side" prescribing, fear of reprimands for "insufficiently intensive therapy" and traditions - coupled with maternal pro-antibiotic attitude. We showed that diagnostic (on-the-spot test for GABHS) and treatment (saline inhalation instructions) interventions reduce maternal uncertainty regarding the nature of the illness and results in a drastic reduction of antibiotic use.

\section{Disclosure}

The authors have no conflict of interests of any kind. No potential, perceived, or real grants had been received. The correspondent author wrote the first draft of the manuscript and no honorarium, grant, or other form of payment was given to anyone to produce the manuscript.

\section{References}

[1] Sabuncu E, David J, Bernède-Bauduin C, Significant reduction of antibiotic use in the community after a nationwide campaign in France, 2002-2007. PLoS Med. 2009 Jun 2; 6(6):e1000084.

[2] Windfuhr JP, Toepfner N, Steffen G, Waldfahrer F, Berner R Clinical practice guideline: tonsillitis I. Diagnostics and nonsurgical management. Eur Arch Otorhinolaryngol. 2016; 273(4):973-87.

[3] McCullough $\mathrm{AR}^{1}$, Pollack $\mathrm{AJ}^{2}$, Plejdrup Hansen $\mathrm{M}^{3}$, Glasziou $\mathrm{PP}^{4}$, Looke $\mathrm{DF}^{5}$, Britt $\mathrm{HC}^{6}$, Del Mar CB. Antibiotics for acute respiratory infections in general practice: comparison of prescribing rates with guideline recommendations. Med J Aust. 2017; 17; 207 (2):65-69.

[4] A. A. Baranov, V. Tatochenko, M. Bakradze (editors). The child with fever. Protocols of diagnosis and treatvment. $3^{\mathrm{d}}$ ed. Moscow, Pediatr, 2017.
[5] NICE. Respiratory tract infections-antibiotic prescribing. Prescribing of antibiotics for self-limiting respiratory tract infections in adults and children in primary care. Clinical Guideline 69, 2008.

[6] Ivanovska V, Hek K, Mantel Teeuwisse AK, Leufkens H, Nielen M, van Dijk L. " Antibiotic prescribing for children in primary care and adherence to treatment guidelines" J. Antimicrob Chemother. 2016 Jun; 71 (6):1707-14.

[7] Ghosh R, Rossner P, Honkova K, Dostal M, Sram RJ, HertzPicciotto I. Air pollution and childhood bronchitis: Interaction with xenobiotic, immune regulatory and DNA repair genes. Environ Int. 2016; 87: 94-100.

[8] Tatochenko V. K. Diseases of respiratory organs in children (new ed). Moscow, Pediatr 2015. - 395 p.

[9] Kuchar E, Miśkiewicz K, Szenborn L, Kurpas D Adv Exp Med Biol. Respiratory tract infections in children in primary healthcare in Poland. 2015; 835: 53-9.

[10] Gardiner SJ, Gavranich JB, Chang AB. Antibiotics for community-acquired lower respiratory tract infections secondary to Mycoplasma pneumoniae in children. Cochrane Database Syst Rev. 2015 Jan 8.

[11] Farley R, Spurling GKP, Eriksson L, Del Mar CB: Antibiotics for bronchiolitis in children under two years of age. Cochrane Database Syst Rev. 2014 Oct 9;(10).

[12] Shulman ST, Bisno AL, Clegg HW, Gerber MA, Kaplan EL, Lee $\mathrm{G}$, et al. Clinical practice guideline for the diagnosis and management of group a streptococcal pharyngitis: 2012 update by the infectious diseases society of America. Clin Infect Dis. 2012; 55 (10):1279-1282.

[13] Ralston SL, Lieberthal AS, Meissner HC, et al. Clinical practice guideline: The diagnosis, management, and prevention of bronchiolitis. Pediatrics. 2014; 134 (5):e1474502 .

[14] Hersh AL Shapiro DJ, Pavia AT, Shah SS.. Antibiotic prescribing in ambulatory pediatrics in the United States. Pediatrics. 2011; 128 (6): 1053-61.

[15] Fleming-Dutra KE, Hersh AL, Shapiro DJ, Bartoces M, Enns EA, File TM Jr et al. Prevalence of inappropriate antibiotic prescriptions among US ambulatory care visits, 2010-2011. JAMA. 2016; 315(17): 1864-73.

[16] Zhukova O. V., Kononova S. V., Konyshkina T. M. [Distribution of expenditures to pharmacotherapy of acute obstructive bronchitis in children under the practical clinical conditions. A pharmacoeconomic study - Rus,]. Farmateka, Moscow). 2011; 18 (231): 63-67.

[17] Kulitchenko T. V., Baibarina E. N., Baranov A. A., Namazova-Baranova L. S. Piskunova S. G., Besedina E. A., et al. [Audit of pediatric hospital services in the regions of Russian Federation - Rus] Vestnik RAMN. 2016. NO 71 (3). 214-223.

[18] Darmanjan A. S. [Practical use of modern diagnostic methods of the streptococcal infection - Rus.]Pediatric pharmmacology. 2013; 10 (5): 97-100.

[19] E. Cherkasova, T. Kuznetsova. [Analysis of antibacterial treatment tactics of acute tonsillitis in children based on a questionnaire survey Russ. pediatric J. 2015; 18(5): 4-5. 
[20] Mandelberg A, Amirav I.; Hypertonic saline or high volume normal saline for viral bronchiolitis: mechanisms and rationale; Pediatr Pulmonol; 2010; NO 45: 36-40.

[21] McIsaac W. J. et al. Empirical validation of guidelines for the management of pharyngitis in children and adults// JAMA/ 2004; 291: 1587-95.

[22] Sukhorukova DN, Kuznetsova TA. [The treatment of an acute bronchiolitis in children in the context of a district covered by child health care.] Russ. pediatric J. 2017; 20(5): 329-333.
[23] Dronov I, A., Geppe N. A. MalakhovA. B., Kondjurina E, G, $\mathrm{m}$ Tatarenko U. A., Tjuteva E, J., Michalev E. V. [Knowledge of pediatricians about antibacterial therapy - the fi rst results of a interregional study]. Pediatric gastroenterology 2014; 11: 14-18. 